\title{
Poincaré Invariant Three-Body Scattering at Intermediate Energies
}

\author{
Ch. Elster ${ }^{1, a}$, T. Lin $^{2}$, W.N. Polyzou ${ }^{3}$, W. Glöckle ${ }^{4}$ \\ 1 Institute of Nuclear and Particle Physics, and Department of Physics, \\ Ohio University, Athens, Ohio 45701, USA \\ 2 Harvard-Smithsonian Center for Astrophysics, Cambridge, MA 02138, USA \\ 3 Department of Physics and Astronomy, The University of Iowa, Iowa City, IA 52242, USA \\ 4 Institut für Theoretische Physik II, Ruhr-Universität Bochum, \\ D-44780 Bochum, Germany
}

\begin{abstract}
Relativistic Faddeev equations for three-body scattering are solved at arbitrary energies in terms of momentum vectors without employing a partial wave decomposition. Relativistic invariance is incorporated within the framework of Poincaré invariant quantum mechanics. Based on a Malfliet-Tjon interaction, observables for elastic and breakup scattering are calculated and compared to non-relativistic ones. The convergence of the Faddeev multiple scattering series is investigated at higher energies.
\end{abstract}

\section{Introduction}

A consistent treatment of intermediate energy reactions requires a Poincaré symmetric quantum theory [1]. In addition, the standard partial wave decomposition, successfully applied below the pion-production threshold [2], is no longer an adequate numerical scheme due to the proliferation of the number of partial waves. Thus, the intermediate energy regime is a new territory for few-body calculations, which waits to be explored.

This work addresses two aspects in this list of challenges: exact Poincaré invariance and calculations using vector variables instead of partial waves. In Ref. [3] the non-relativistic Faddeev equations were solved directly as function of vector variables for scattering at intermediate energies. A key advantage of this formulation lies in its applicability at higher energies, where the number of partial waves proliferates. The Faddeev equation, based on a Poincaré invariant mass operator, has been formulated in detail in [4] and has both kinematical and dynamical differences with respect to the corresponding non-relativistic equation.

\section{Theoretical Aspects}

The formulation of the theory is given in a representation of Poincaré invariant quantum mechanics where the

\footnotetext{
${ }^{a}$ e-mail: elster@ohiou.edu
}

interactions are invariant with respect to kinematic translations and rotations [5]. The model Hilbert space is a threenucleon Hilbert space (thus not allowing for absorptive processes). The method introduces the $\mathrm{NN}$ interactions in the unitary representation of the Poincare group and allows to input e.g. high-precision NN interactions in a way that reproduces the measured two-body observables. However in this study we use a simpler interaction consisting of a superposition of an attractive and a repulsive Yukawa interaction with parameters chosen such that a bound state at $E_{d}=-2.23 \mathrm{MeV}$ is supported [4]. Poincaré invariance and $S$-matrix cluster properties dictate how the two-body interactions must be embedded in the three-body dynamical generators. Scattering observables are calculated using Faddeev equations formulated with the mass Casimir operator (rest Hamiltonian) constructed from these generators.

To obtain a valid estimate of the size of relativistic effects, it is important that the interactions employed in the relativistic and non-relativistic calculations are phase-shift equivalent. We follow the suggestion by Coester, Piper, and Serduke (CPS) and construct a phase equivalent interaction from a non-relativistic $2 \mathrm{~N}$ interaction [6] by adding the interaction to the square of the mass operator. In this CPS method the relativistic interaction can not be analytically calculated from the non-relativistic one. However, there is a simple analytic connection between the relativistic and non-relativistic two-body t-matrices

$t_{r e}\left(\mathbf{p}, \mathbf{p}^{\prime} ; 2 E_{p}^{r e l}\right)=\frac{2 m}{\sqrt{m^{2}+p^{2}}+\sqrt{m^{2}+p^{\prime 2}}} t_{n r}\left(\mathbf{p}, \mathbf{p}^{\prime} ; 2 E_{p}^{n r}\right)$,

This is an Open Access article distributed under the terms of the Creative Commons Attribution-Noncommercial License 3.0, which permits unrestricted use, distribution, and reproduction in any noncommercial medium, provided the original work is properly cited. 

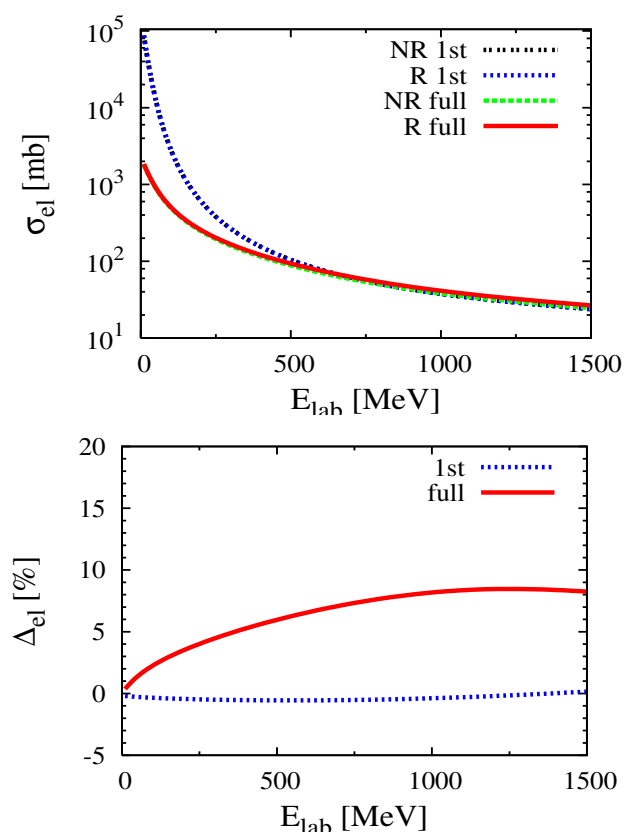

Fig. 1. The total c.m. cross section for elastic calculated from a Malfliet-Tjon type potential as function of the projectile kinetic laboratory energy. The labels ' $R$ ' ('NR') stand for relativistic (non-relativistic) calculations. The Faddeev calculations in the first order in $t$ are marked with '1st', the converged full Faddeev calculations with 'full'. To show the difference, the percentage difference between the relativistic and corresponding nonrelativistic calculations is displayed in the lower panel.

where $2 E_{p}^{r e l}=2 \sqrt{m^{2}+p^{2}}$ and $2 E_{p}^{n r}=\frac{p^{2}}{m}+2 m$. This relativistic two-body t-matrix $t_{r e}\left(\mathbf{p}, \mathbf{p}^{\prime} ; 2 E_{p}^{r e l}\right)$ is scattering equivalent to the non-relativistic one at the same relative momentum p [7].

This t-matrix is the input for the Poincare invariant transition amplitude of the $2 \mathrm{~N}$ subsystem embedded in the three-particle Hilbert space obtained via a first resolvent method as layed out in Ref. [4].

By construction, differences in the relativistic and nonrelativistic calculations first appear in the three-body calculations. Those differences are in the choice of kinematic variables (Jacobi momenta are constructed using Lorentz boosts rather then Galilean boosts) and in the embedding of the two-body interactions in the three-body problem, which is a consequence of the non-linear relation between the two and three-body mass operators. These differences modify the permutation operators and the off-shell properties of the kernel of the Faddeev equations [9].

\section{Selected Results}

In Figs. 1 and 2 the total cross sections for elastic and breakup cross sections are displayed as function of the projectile kinetic energy up to $1.5 \mathrm{GeV}$ obtained from our fully converged relativistic Faddeev calculation as well as the one obtained from the first-order term, $T^{1 s t}=t P$, with $P$ being the permutation operator for three identical particles.
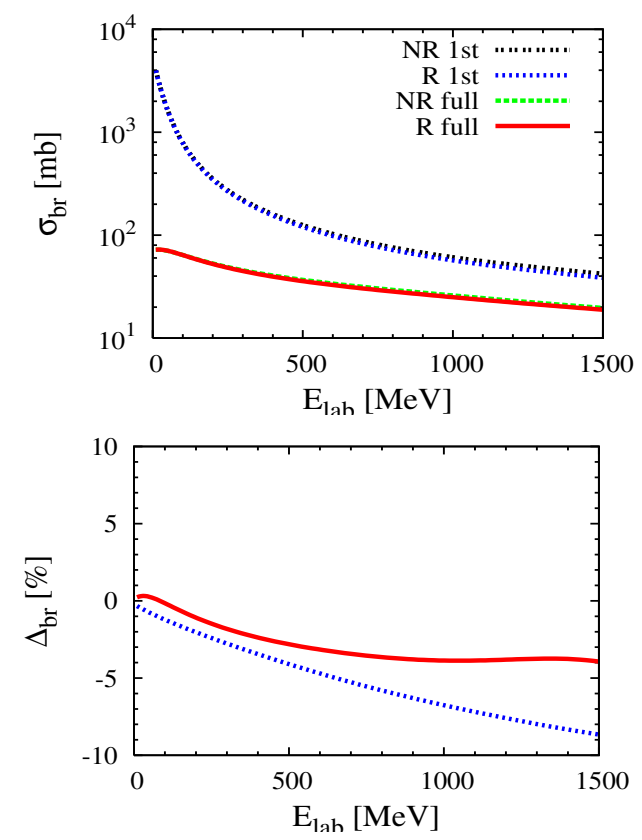

Fig. 2. The total c.m. cross section for breakup scattering calculated from a Malfliet-Tjon type potential as function of the projectile kinetic laboratory energy. The labels 'R' ('NR') stand for relativistic (non-relativistic) calculations. The Faddeev calculations in the first order in $t$ are marked with '1st', the converged full Faddeev calculations with 'full'. To show the difference, the percentage difference between the relativistic and corresponding non-relativistic calculations is displayed in the lower panel.

It is obvious that, especially for energies below $300 \mathrm{MeV}$, the contribution of rescattering terms is huge. However, for extracting the size of relativistic effect, it is more useful to consider the relative difference between the relativistic and non-relativistic calculations. In first order, there is essentially no effect in the total elastic cross section, which is consistent with the observation that the relativistic two-body $t$-matrix is constructed to be phase-shift equivalent to the non-relativistic one. The same comparison with fully converged Faddeev calculations indicates that relativistic effects in the three-body problem increase the total cross section for elastic scattering with increasing energy, whereas it is slightly reduced in the total breakup cross section.

Furthermore, we want to investigate the convergence of the multiple scattering series as function of the projectile laboratory kinetic energy. One might expect that with increasing energy only a few terms in the multiple scattering series are sufficient for a converged result. Our converged relativistic Faddeev calculations now allow a detailed study. This is of particular interest, since recently relativistic calculations in the energy regime around $1 \mathrm{GeV}$ have been published $[10,11]$, which are carried out in a multiple scattering expansion of the Faddeev equations up to 2nd order, and which use the off-shell continuation of the experimental $\mathrm{NN}$ amplitudes as two-body input.

The upper panel of Fig. 3 shows the elastic total cross section as function of the order in the multiple scattering 

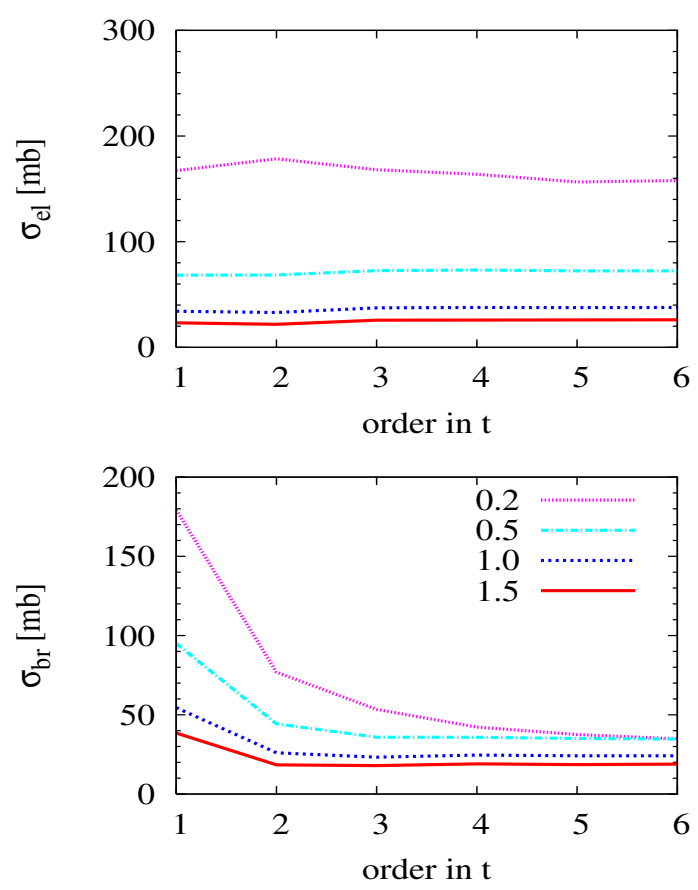

Fig. 3. The total cross section for elastic scattering (top panel) and for breakup reactions (bottom panel) for different projectile laboratory kinetic energies as function of the order in the multiple scattering series. Here the orders are successively added. The values of the projectile laboratory kinetic energies are given in the legends of the bottom panel in units of $\mathrm{GeV}$.

series (the orders are successively summed up the one indicated on the $\mathrm{x}$-axis). Even at $200 \mathrm{MeV}$ there is very little change due to contributions from the 2 nd or higher order rescattering terms. For the higher energies, the 1st order term already captures the essential physics. This is very different for the total breakup cross section, shown in the lower panel of Fig. 3. where for $200 \mathrm{MeV}$ projectile energy the full solution of the Faddeev equation is clearly necessary. For energies of $1 \mathrm{GeV}$ and higher, at least one rescattering contribution (2nd order in the multiple scattering series) is necessary to come close to the full solution.

Considering exclusive breakup reactions, differences between a relativistic and non-relativistic calculation are more pronounced and strongly depend on the configuration. Though our two-body force is simple, we compare to a ${ }^{2} \mathrm{H}(\mathrm{p}, 2 \mathrm{p}) \mathrm{n}$ experiment at $508 \mathrm{MeV}$ [12] to see if our calculation captures essential features of the measurement. Differences in the predictions of our relativistic and nonrelativistic calculations are very pronounced at this energy as can be seen in Fig. 4, which shows selected angle pairs $\theta_{1}-\theta_{2}$ from Ref. [12], which are symmetric around the beam axis. The cross section is plotted against the laboratory kinetic energy of one of the outgoing protons. It is interesting to observe that for smaller angle pairs the relativistic cross sections (RF) are considerably larger than the non-relativistic ones (NRF). For larger angle pairs the situation reverses. It is further noteworthy, that in the configurations of Fig. 4, which are close to quasi-free, rescattering

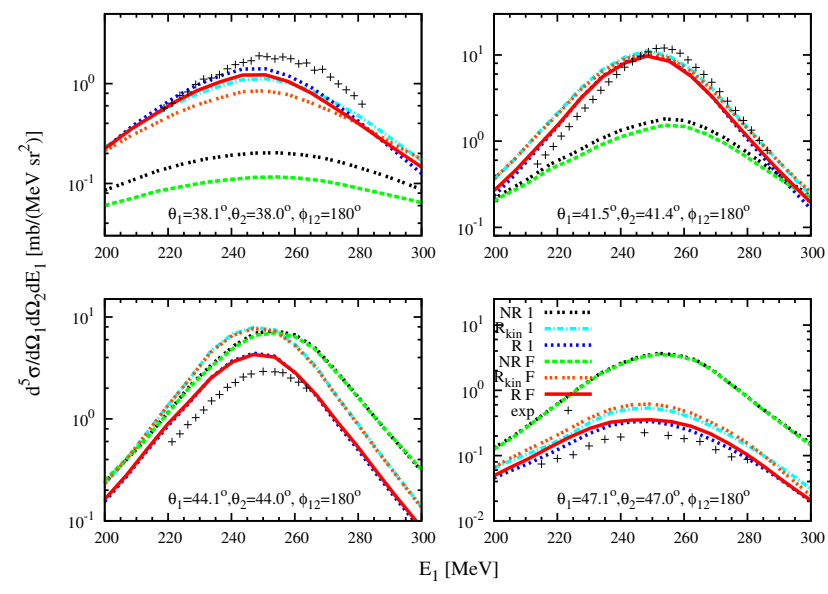

Fig. 4. The exclusive differential cross section for the reaction ${ }^{2} \mathrm{H}(\mathrm{p}, 2 \mathrm{p}) \mathrm{n}$ at $508 \mathrm{MeV}$ laboratory projectile energy for different proton angle pairs $\theta_{1}-\theta_{2}$ symmetric around the beam axis as function of the laboratory kinetic energy of one of the outgoing protons. The meaning of the curves are the same as in Fig. 1, except that here ' 1 ' denotes the 1 st order Faddeev calculation, 'F' the fully converged one. In the curves labeled $R_{\text {kin }}$ only relativistic kinematics is taken into account. The data are taken from Ref. [12].

effects (or equivalently higher order contributions of the Faddeev multiple scattering series) are very small (curves ' 1 ' and ' $F$ ' are almost identical). To show that peak-positions are given by kinematics, we added curves labeled ' $\mathrm{R}_{\text {kin }}$ ', which stands for a non-relativistic calculation in which only kinematics and phase space factors are replaced by the relativistic ones. We want to note that the above comparisons do not involve a non-relativistic limit, instead relativistic and non-relativistic three-body calculations with interactions that are fit to the same two-body data are compared. All of the differences are due to the different ways twobody dynamics is incorporated in the three-body problem.

In Fig. 5 we show the convergence of the Faddeev multiple-scattering series in the differential cross for selected breakup configurations at $508 \mathrm{MeV}$ projectile laboratory kinetic energy. The final configurations are set up for the two outgoing particles being on each side of the incoming beam. In the configurations shown in the top two panels, the two angles $\theta_{1}$ and $\theta_{2}$ with respect to the beam are equal, whereas they are not equal in the two bottom panels. It can be seen that in all cases the multiple scattering series converges quite fast. The fastest convergence, however, occurs in the configuration with $\theta_{1}=41.5^{\circ}, \theta_{2}=41.4^{\circ}$, which is a quasi-free scattering (QFS) configuration. This leads to the conclusion, that quasi-free three-body breakup configurations in the intermediate energy regime may be analyzed by first-order calculations, opening the possibility to study neutron-proton scattering above a $\mathrm{GeV}$. 

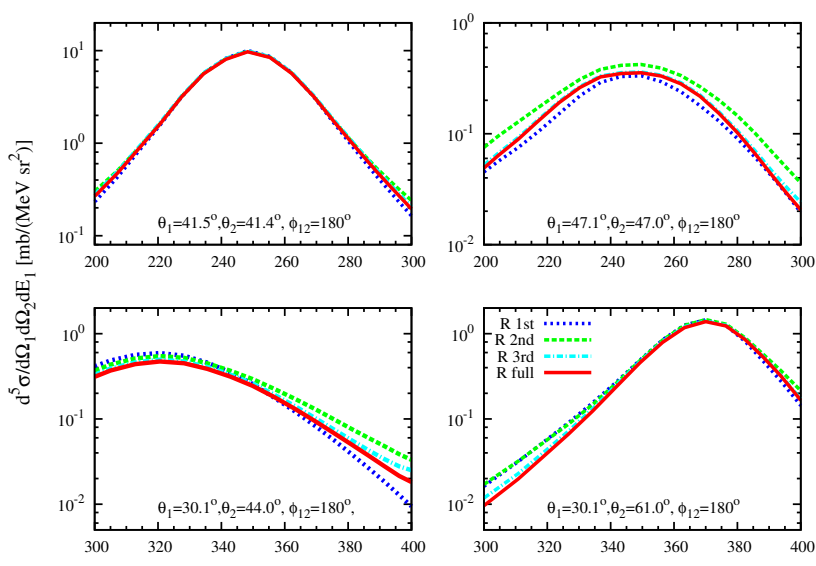

$\mathrm{E}_{1}[\mathrm{MeV}]$

Fig. 5. The differential cross section for exclusive breakup scattering for $508 \mathrm{MeV}$ laboratory projectile kinetic energy as function of the laboratory kinetic energy of one of the outgoing protons. The two outgoing particles are measured on each side of the beam. Upper panel: Configurations with outgoing particle angle pairs $\theta_{1}-\theta_{2}$ symmetric around the beam axis. Lower panel: Configurations with outgoing particle angles $\theta_{1}-\theta_{2}$ asymmetric around the beam. The full Faddeev calculation is indicated by the solid (red) line. The first order calculation is indicated by the dotted (blue) line. Faddeev calculations for the next two orders are added successively the to the first order one, and indicated by the lines given in the legend of the lower left panel.

\section{Summary}

In this work we demonstrate the feasibility of applying Poincaré invariant quantum mechanics to model three-nucleon reactions at energies up to $2 \mathrm{GeV}$. This is an important first step for studying dynamical models of strongly interacting particles in the energy range where sub-nuclear degrees of freedom are thought to be relevant. At these energies the Poincaré invariance of the theory is an essential symmetry. At lower energies non-relativistic quantum mechanical models are powerful tools for understanding the dynamics of strongly interacting nucleons. At higher energies the physics is more complicated, but one can expect that it is still dominated by a manageable number of degrees of freedom. Poincaré invariant quantum mechanics is the only alternative to quantum field theory where it is possible to realize the essential requirements of Poincaré invariance, spectral condition, and cluster properties [13]. It has the advantage that the Faddeev equation provides a mathematically well-defined method for exactly solving the strong interaction dynamics. The Faddeev equation in this framework is more complicated than the corresponding non-relativistic equation, due to the non-linear relation between the mass and energy in relativistic theories, but these difficulties can be overcome [4,7,14]. An important advance that allows these calculations to be extended to energies in the $\mathrm{GeV}$ range is the use of numerical methods based on direct integrations, rather than partial wave expansions. These have been successfully applied to the nonrelativistic three-nucleon problem $[3,17]$. Here we demon- strate that they can also be successfully applied to the relativistic problem, even with its additional complications.

The model presented here involves three nucleons interacting with a spin-independent Malfliet-Tjon [18] type of interaction. It differs from more realistic interactions in that it is spin independent and it does not give a highprecision fit to the two-body scattering data. In addition, the model is for fixed numbers of particles, not allowing pion production, which is an open channel at these energies. While these limitations must be addressed in realistic applications, the three-body Faddeev calculations presented here provide a powerful framework for both testing approximations and for examining the sensitivity of scattering observables to relativistic effects.

In order to investigate relativistic effects, we treat the interaction as if it was determined by fitting the cross section obtained by solving the non-relativistic Lipp-mannSchwinger equation to scattering data. When this is done with a realistic interaction the experimental differential cross section is properly transformed from the laboratory frame to the center of momentum frame before the fit is done. The result of this process is that the computed differential cross section agrees with the fully-relativistic experimental differential cross section in the center of momentum frame as a function of the relative momentum. Thus, even though the two-body scattering observables are computed with a non-relativistic equation, there is nothing non-relativistic about the result. At the two-body level the corresponding relativistic Lippmann-Schwinger equation must be designed to give the same scattering observables. This can be achieved by expressing the relativistic mass operator as a simple function of the non-relativistic center of momentum Hamiltonian $[15,16]$. The important consequence of this is that it does not make sense to relate the relativistic and non-relativistic two-body models using $p / m$ expansions; the prediction of the relativistic and non-relativistic two-body models are identical. Real differences in the dynamics appear when the two-body dynamical operators are used to formulate the three-body dynamics. How this must be done in the two and three-body cases is dictated, up to three-body interactions, by cluster properties. The Faddeev equation for the relativistic and non-relativistic system have identical operators forms. The permutation operators, two-body transition operators and free resolvents that are input to the Faddeev equation have different forms in the relativistic and non-relativistic equations. These differences are responsible for differences in the relativistic and non-relativistic three-body calculations.

The calculations presented here have a number of consequences. The most important result was a demonstration that direct integration methods can be successfully applied to extend the energy range for converged solutions to Faddeev equations to intermediate energies. Our estimates of the number of partial waves needed for calculations at different energies suggest that it is not currently practical to extend existing partial wave calculations beyond a few hundred $\mathrm{MeV}$, while in this paper we have demonstrated convergence of the direct integration methods for laboratory energies up to $2 \mathrm{GeV}$. 
$19^{\text {th }}$ International IUPAP Conference on Few-Body Problems in Physics

While our model interaction is not realistic, when we compared the results of our calculations to relativistic calculations at $200 \mathrm{MeV}$ that have been performed with realistic interactions [19-21] in a partial wave basis, we found that the qualitative features of the realistic models are reproduced in our simple model. This suggests that some of the conclusions derived from our model should be applicable to models with realistic interactions. However, we demonstrated the need for a relativistic description of fewnucleon dynamics in the intermediate energy range and show that the problem is amenable to a numerically exact solution, using direct integration, for laboratory energies up to $2 \mathrm{GeV}$. Obviously extensions to include spin-depend interactions, meson channels, and interactions that are fit to higher energy data will be needed for realistic applications.

\section{Acknowledgments}

This work was performed in part under the auspices of the U. S. Department of Energy under contract No. DE-FG0293ER40756 with Ohio University and under contract No. DE-FG02-86ER40286 with the University of Iowa.

We thank the Ohio Supercomputer Center (OSC) for the use of their facilities under grant PHS206.

\section{References}

1. E.P. Wigner, Ann. Math. C 40, 149 (1939).

2. W. Glöckle, H. Witała, D. Hüber, H. Kamada, J. Golak, Phys. Rep. 274, 107 (1996).

3. H. Liu, Ch. Elster, W. Glöckle, Phys. Rev. C72, 054003 (2005).

4. T. Lin, Ch. Elster, W. N. Polyzou, W. Glöckle, Phys. Rev. C76, 014010 (2007).

5. F. Coester, Helv. Phys. Acta. 38, 7 (1965).

6. F. Coester, S.C. Piper, and F.J.D. Serduke, Phys. Rev. C11, 1 (1975).

7. B. D. Keister and W. N. Polyzou, Phys. Rev. C73, 014005 (2006).

8. T. Lin, Ch. Elster, W. N. Polyzou, W. Glöckle, Phys. Lett. B 660, 345 (2008).

9. T. Lin, Ch. Elster, W. N. Polyzou, H. Witała, W. Glöckle, Phys. Rev. C78, 024002 (2008).

10. N. B. Ladygina, Eur. Phys. J. A 42, 91 (2009).

11. N. B. Ladygina and A. V. Shebeko, Few Body Syst. 33, 49 (2003)

12. V. Punjabi et al., Phys. Rev. C38, 2728 (1988).

13. Bert Schroer, see arxiv:hep-th/0405105 and arxiv:hepth/0711.4600.

14. H. Kamada and W. Glóckle Phys. Lett. B655,119(2007).

15. F. Coester, S.C. Pieper, F.J.D. Serduke, Phys. Rev. C11, 1 (1975).

16. B.D. Keister and W.N. Polyzou Advances in Nuclear Physics, Volume 20, Ed. J. W. Negele and E. W. Vogt, Plenum Press 1991.

17. H. Liu, Ch. Elster and W. Glöckle, Few Body Syst. 33, 241 (2003)
18. R. A. Malfliet and J. A. Tjon Nucl. Phys. A127,161(1969).

19. H. Witała, J. Golak, W. Glöckle, H. Kamada, Phys. Rev. C71, 054001 (2005).

20. H. Witała, J. Golak, R. Skibiński, Phys. Lett. B 634, 374 (2006).

21. H. Witała, R. Skibiński, J. Golak, Eur. Phys. J. A 29, 141 (2006). 